\title{
Psychological Aspects of the COVID-19 Pandemic
}

\author{
Carlos Blanco, M.D., Ph.D. ${ }^{7}$ (i), Melanie M. Wall, Ph.D. ${ }^{2}$, and Mark Olfson, M.D., M.P.H. ${ }^{2}$
}

Check for updates

'Division of Epidemiology, Services and Prevention Research, National Institute on Drug Abuse Bethesda, MD, USA; ${ }^{2}$ Department of Psychiatry, New York State Psychiatric Institute/Columbia UniversityNew York, NY, USA.

$J$ Gen Intern Med 35(9):2757-9

DOI: $10.1007 / \mathrm{s} 11606-020-05955-3$

(c) Society of General Internal Medicine (This is a U.S. government work and not under copyright protection in the U.S.; foreign copyright protection may apply) 2020

$\mathrm{D}$ iagnoses of coronavirus disease 2019 (COVID-19) in close to 200 countries have led the World Health Organization to declare it a global pandemic. To date, most actions related to COVID-19 have understandably focused on containing or mitigating the spread of the virus, testing new treatments, and seeking to develop vaccines. Much less attention has been directed towards the psychological consequences associated with the pandemic and the measures considered or adopted to combat its life-threatening consequences, even as distress has increased with growing numbers of individuals testing positive, seeing loved ones die, and with uncertainty about when and how a return to previous ways of life might be possible. We outline some potential adverse psychological consequences and offer suggestions for their prevention or mitigation.

\section{INCREASED PREVALENCE OF PSYCHIATRIC DISORDERS AND SYMPTOMS}

Experience from previous disasters suggests that the pandemic is likely to be associated with increased prevalence of psychiatric disorders and clinical symptoms, ${ }^{1}$ although responses may vary by population and local prevalence of the disease. In prior crisis, restricted access to basic necessities (such as food, protective equipment, and medical care) and risk of death, all of which have occurred during the pandemic, have been associated with increased incidence of psychiatric disorders and psychological distress. ${ }^{2}$ Racial and ethnic minorities and those fighting on the front lines appear to be at increased risk of the psychological consequences of natural disasters. ${ }^{2}$

Received April 8, 2020

Revised May 5, 2020

Accepted June 1, 2020

Published online June 15, 2020

\section{EFFECT OF QUARANTINES AND POTENTIAL FOR HEIGHTENED PHYSICAL ILLNESS CONCERN}

Some of the public health measures that may be necessary to contain the spread of the pandemic, such as quarantines and social distancing, may further increase the risk of adverse psychological consequences. A recent review revealed that these risks are greater with longer quarantine periods, and when there are infection fears, frustration, boredom, inadequate supplies, inadequate information, and financial loss. ${ }^{3}$ Even in the absence of formal quarantines, appeals for social and physical distancing may result in increased loneliness and social isolation for many, particularly those most vulnerable, which can further increase risks to physical and psychological health. ${ }^{4}$ Stigmatization of populations perceived as responsible for spreading the virus may arise as an unintended consequence of initiatives enacted to slow or eliminate transmission, such as sealing off borders or restricting travel, and may persist after those policies are relaxed or fully lifted. ${ }^{3}$ Furthermore, because COVID-19 is associated with physical symptoms, some individuals may develop heightened somatic illness concern (i.e., hypochondriasis) and experience physical symptoms that may mimic or be perceived as symptoms of a COVID-19 infection.

\section{PREVENTION AND MITIGATION STRATEGIES}

A number of steps can be taken to address the psychological consequences of the pandemic. First, because uncertainty about future danger is at the core of anxiety, prior experience suggests that an essential first step involves provision of timely, accurate, and contextualized information by scientists, public health officials, and the media that is easy to understand by the general population. ${ }^{3,5}$ In the initial stages of the pandemic, the information provided to the lay public in most countries focused mostly on the number of cumulative cases (or deaths) or the number of new cases detected from the previous day, but seldom differentiated among different levels of severity (e.g., positive for the virus but asymptomatic; having mild symptoms that required only social isolation to avoid the spread of the virus; and how many needed hospital-based or intensive unit care). Stratifying reports by readily discernible risk factors, such as age or presence of pre-existing comorbid conditions, which has been done more recently, ${ }^{5}$ can also facilitate contextualizing and explaining results to the general public. As the 
pandemic continues to evolve, public officials and other policy makers will have key roles in providing information about the expected outcome of different approaches (e.g., different strategies for lifting the lockdowns), promising treatments or vaccine developments and changes in the incidence and prevalence of infections stratified by relevant factors, such as populations at risk and outcome of the infection (e.g., hospitalization, recovery, or death).

A second step to help reduce anxiety involves provision of the explicit rationale for the clinical and public health approaches taken. ${ }^{3}$ Inter-country and local variations in measures implemented to date have not always been accompanied by information on the scientific support for those strategies or their expected duration. For example, if measures adopted or proposed are based on mathematical models of the course of the pandemic, model developers should disclose the assumptions underlying each model, the evidence supporting their assumptions, and the projected outcomes of competing strategies. Examining those assumptions maybe particularly important when jointly modeling effects of different strategies (e.g., length of the lockdown or phased lifting of social distancing restrictions) on the course of the pandemic and economic outcomes. Model developers should also deposit the code for their models in an open-code repository such as Github to ensure their reproducibility and to facilitate adaptation for cities or countries by appropriately modifying model parameters. Making this information available would help scientists and the lay public understand the basis for choices among competing strategies to balance public health safety, limitations of individual liberties such as restrictions on movement or the size of gatherings, and economic outcomes.

Prior experience suggests that it is also important to ensure access to mental health care, personalize the level and type of interventions for those who need them, and foster integration psychological and general medical care. At the population level, we can expect variability in the psychological responses to the pandemic. ${ }^{1-3}$ Based on findings from previous studies of catastrophes, we should expect that most people will experience no or mild psychological symptoms and that provision of the information suggested above will be sufficient to address their concerns. ${ }^{1,2}$ Individuals with mild to moderate distress may benefit from low intensity interventions, such as meditation or relaxation techniques. For individuals with more severe psychological distress or bona fide psychiatric disorders, including substance use disorders, collaborative care models could help ensure timely access to treatment for those who need it in an efficient manner. ${ }^{6}$ In these models, patients are initially seen by less specialized clinicians who have access to consultation from specialists who focus on managing complex cases (e.g., those with multiple co-occurring disorders) and those who have not responded to initial treatment approaches. Clinicians, in discussion with patients and in accordance with guidance from public health officials and health system leaders, will need to decide when care needs to be provided in person and when it can be provided through other means, such as via telehealth, to limit unnecessary travel and exposure to physical contact.

Managing patients with hypochondriasis may be particularly difficult. Denying testing to those for whom it is indicated would be clearly inappropriate from the clinical, ethical, and public health point of view, while testing those without proper indication could exacerbate patients' anxiety and potentially deprive testing to others for whom it is medically indicated. Establishing clear guidelines, based on the existing evidence, of who should be tested could help individual clinicians make those difficult decisions. Frameworks for allocation of ventilators and critical care beds during the pandemic, as well as the process to develop those frameworks, can serve as models for guideline development. Guidelines, developed in collaboration by diverse citizen groups, ethicists, and disaster experts, could be modified as the epidemiology of COVID-19 is better understood and testing becomes more widely available. ${ }^{7}$

Although there may be a need to limit physical contact, there is also a clear need to foster social support and promote solidarity and social cohesion. Social support is a powerful predictor of physical and emotional health. ${ }^{4}$ Solidarity, social cohesion, and altruism will be necessary to withstand the individual and collective sacrifices necessary to combat the COVID-19 pandemic and its aftermath and to avoid scapegoating of particular segments of the population. ${ }^{1,3}$ Adhering to public health measures to decrease virus transmission, such as maintaining appropriate physical distance and using face coverings, are important manifestations of solidarity and care for others. Frontline and essential workers including health workers, first responders, and providers of services such as food and other basic needs that risk their lives to maintain the functioning of society constitute important examples of altruism. ${ }^{3}$ Public recognition of their sacrifices could also foster social cohesion. In the short term, digital technologies, including videoconferencing and social media, can foster solidarity and cohesion by helping bridge social distance and facilitating connections with friends, family, and coworkers. $^{3}$ In the longer term, there may be a need for societal debate regarding what technologies, social structures, and values best promote resilience in the event of future pandemics or other natural disasters.

\section{CONCLUSION}

The COVID-19 pandemic presents new challenges to public health and the regular functioning of many aspects to society, including the psychological functioning of individuals and groups. As we learn how best to respond these challenges, experience from previous crises suggests populations at increased risk and approaches to help minimize the psychological impact of the pandemic.

Corresponding Author: Carlos Blanco, M.D., Ph.D.; Division of Epidemiology, Services and Prevention Research, National Institute on Drug Abuse Bethesda, MD, USA (e-mail: carlos.blanco2@nih.gov). 


\section{Compliance with Ethical Standards:}

Conflict of Interest: The authors declare that they do not have a conflict of interest.

Disclaimer: The views and opinions expressed in this report are those of the authors and should not be construed to represent the views of the National Institute on Drug Abuse, the National Institutes of Health or any US government agency.

\section{REFERENCES}

1. Beaglehole B, Mulder RT, Frampton CM, Boden JM, Newton-Howes G, Bell CJ. Psychological distress and psychiatric disorder after natural disasters: systematic review and meta-analysis. Br J Psychiatry. 2018;213(6):716-722. doi: https://doi.org/10.1192/bjp.2018.210.

2. Chan CS, Rhodes JE. Measuring exposure in Hurricane Katrina: a metaanalysis and an integrative data analysis. PLoS One. 2014;9(4):e92899. doi: https://doi.org/10.1371/journal.pone.0092899. eCollection 2014.
3. Brooks SK, Webster RK, Smith LE, et al. The psychological impact of quarantine and how to reduce it: rapid review of the evidence. Lancet. 2020

4. National Academies of Sciences, Engineering, and Medicine 2020. Social Isolation and Loneliness in Older Adults: Opportunities for the Health Care System. Washington, DC: The National Academies Press.

5. Garg S, Kim L, Whitaker M, O'Halloran A, et al. Hospitalization Rates and Characteristics of Patients Hospitalized with Laboratory-Confirmed Coronavirus Disease 2019 - COVID-NET, 14 States, March 1-30, 2020. MMWR Morb Mortal Wkly Rep. 2020;69(15):458-464.

6. Archer J, Bower P, Gilbody S, et al. Collaborative care for depression and anxiety problems. Cochrane Database Syst Rev. 2012;10:CD006525.

7. White DB, Lo B. A Framework for Rationing Ventilators and Critical Care Beds During the COVID-19 Pandemic. JAMA. 2020. doi: https://doi.org/ $10.1001 /$ jama.2020.5046

Publisher's Note: Springer Nature remains neutral with regard to jurisdictional claims in published maps and institutional affiliations. 